\title{
A carrier-assisted ChIP-seq method for estrogen receptor-chromatin interactions from breast cancer core needle biopsy samples
}

\author{
Wilbert Zwart ${ }^{1,5}$, Rutger Koornstra ${ }^{1}$, Jelle Wesseling ${ }^{1,2}$, Emiel Rutgers ${ }^{3}$, Sabine Linn ${ }^{1,4}$ and Jason S Carroll ${ }^{5,6^{*}}$
}

\begin{abstract}
Background: The Estrogen Receptor alpha (ERa) is the key transcriptional regulator in luminal breast cancer and is therefore the main target for adjuvant treatment of this subtype. Luminal gene signatures are dictated by the transcriptional capacities of $\mathrm{ERa}$, which are a direct consequence of the receptors binding preference at specific sites on the chromatin. The identification of ERa binding signatures on a genome-wide level has greatly enhanced our understanding of Estrogen Receptor biology in cell lines and tumours, but the technique has its limitations with respect to its applicability in limited amounts of tumour tissue.

Results: Here, we present a refinement of the ChIP-seq procedures to enable transcription factor mapping on limited amounts of tissue culture cells as well as from a limited amount of tumor tissue derived from core needle biopsies. Our approach uses a carrier that can be removed prior to DNA amplification and sequencing.

Conclusion: We illustrate the applicability of this refined technology by mapping the ERa genome-wide chromatin binding landscape in core needle biopsy material from primary breast tumours. With this, our refined technology permits for a high-resolution transcription factor mapping even from clinical samples.
\end{abstract}

\section{Background}

Breast cancer is the most common diagnosed malignancy in women, with over 1.4 million new cases worldwide annually [1]. $75 \%$ of all breast cancers are from the luminal subtype [2] for which tumour growth is thought to be dependent on the activity of the Estrogen Receptor alpha (ER $\alpha)$. This growth-dependency renders this pathway the main target for adjuvant endocrine treatment in luminal breast cancer.

The physiological behaviour of the ER $\alpha$ involves binding of the receptor to its natural ligand estradiol, after which the receptor associates to the chromatin, recruits its coregulators and alters the transcriptional activity of responsive genes, leading to increased cell proliferation and tumour growth. In endocrine treatment of breast cancer, the activation of the receptor can be inhibited through multiple ways, each of which resulting in an

\footnotetext{
* Correspondence: Jason.Carroll@cruk.cam.ac.uk

${ }^{5}$ Cancer Research UK, Cambridge Research Institute, Li Ka Shing Centre, Robinson Way, Cambridge CB2 ORE, UK

${ }^{6}$ Department of Oncology, University of Cambridge, Cambridge CB2 OXY, UK Full list of author information is available at the end of the article
}

inhibition of ER $\alpha$-driven cell proliferation. Even though these endocrine intervention therapies greatly increase the disease-free survival and overall survival of breast cancer patients [3], resistance to treatment is commonly observed. This resistance can be mediated through a multitude of different mechanisms, including differential expression of kinases $[4,5]$, coregulators $[3,6]$ and transmembrane receptors $[7,8]$.

Importantly, it is becoming apparent that mechanisms of resistance may result from intrinsic effects on $E R \alpha /$ chromatin interactions, as shown both in cell lines $[9,10]$ and in tumour samples [11]. Since the ER $\alpha /$ chromatin interactome directly determines the estradiol-mediated effects on gene expression [12], distinct binding patterns may be a key-defining factor for deviating gene expression patterns as well [13]. A major hurdle to assay such transcription factor/chromatin interactions, is the frequently very limited starting material due to the minimal amount of tissue that is obtained during diagnostic work-up.

Recently approaches have been described to map histone modifications in a limited amount of starting

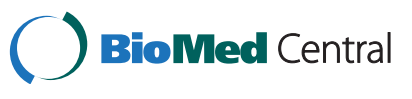


material [14], but no such protocol has been described for transcription factors. Similarly, new methods for amplification of limited material have been described [15], but these assume that sufficient DNA was recovered from the ChIP to enable amplification in the first place. One of the major technical limitations has been enriching sufficient DNA (via ChIP) from limited starting material. This problem has been addressed using 'carrier chromatin' [16], a strategy that is not amenable to global sequencing approaches. Here, we describe a procedure to enhance transcription factor ChIP-seq by incorporating a modified carrier method to enable ChIPseq from limited starting material. We apply this method to map the $E R \alpha /$ chromatin interactome in as few as 10,000 tissue culture cells and apply this technology to map the ER $\alpha$ chromatin binding landscape in core needle biopsy samples from breast cancer patients.

\section{Results and discussion}

\section{Carrier selection for small cell number ChIPs}

To optimize the Chromatin Immunoprecipitation (ChIP) procedure from a limited amount of cells, we utilized the well-characterized (ER $\alpha$-positive and Progesterone Receptor-positive) breast cancer cell line MCF7. MCF7 cells have been widely applied for ChIP-seq applications, and multiple datasets are publicly available to be used as a reference. Proliferating MCF7 cells from a sub-confluent $10 \mathrm{~cm}$ dish were harvested, after which the total amount of cells were counted. The lysate from 10,000 cells was used for ChIP. Initially a focused ER $\alpha$ ChIP-real time PCR approach was taken, using the well-studied pS2 promoter region. ER $\alpha$ ChIP was performed using standard methods [17], but in the absence of carrier, no enrichment from the ChIP sample was observed (Figure 1A). In addition, two independent carriers were tested: glycogen and mRNA/ histones. The rationale was to test carriers that were either inert (glycogen; not having any effect on the chromatin nor sequencing procedure) or had a composition that could be removed from the sample (mRNA and histones; to be removed by RNAse A and Proteinase K). The carriers were added to the lysate mixture before antibodyconjugate addition. Glycogen proved to have a small, but not significant increase in ChIP efficiency, when using the pS2 promoter region. The addition of recombinant histones and random human mRNA led to a significant increase of ER $\alpha$ signal at the pS2 promoter (Figure 1A). This enhanced enrichment was achieved both by an increased ChIP efficiency for specific signal as well as a decrease of the nonspecific background binding (Additional file 1: Figure S1). Combining the two carrier procedures resulted in a decreased signal as compared to only adding $\mathrm{mRNA} /$ histones.

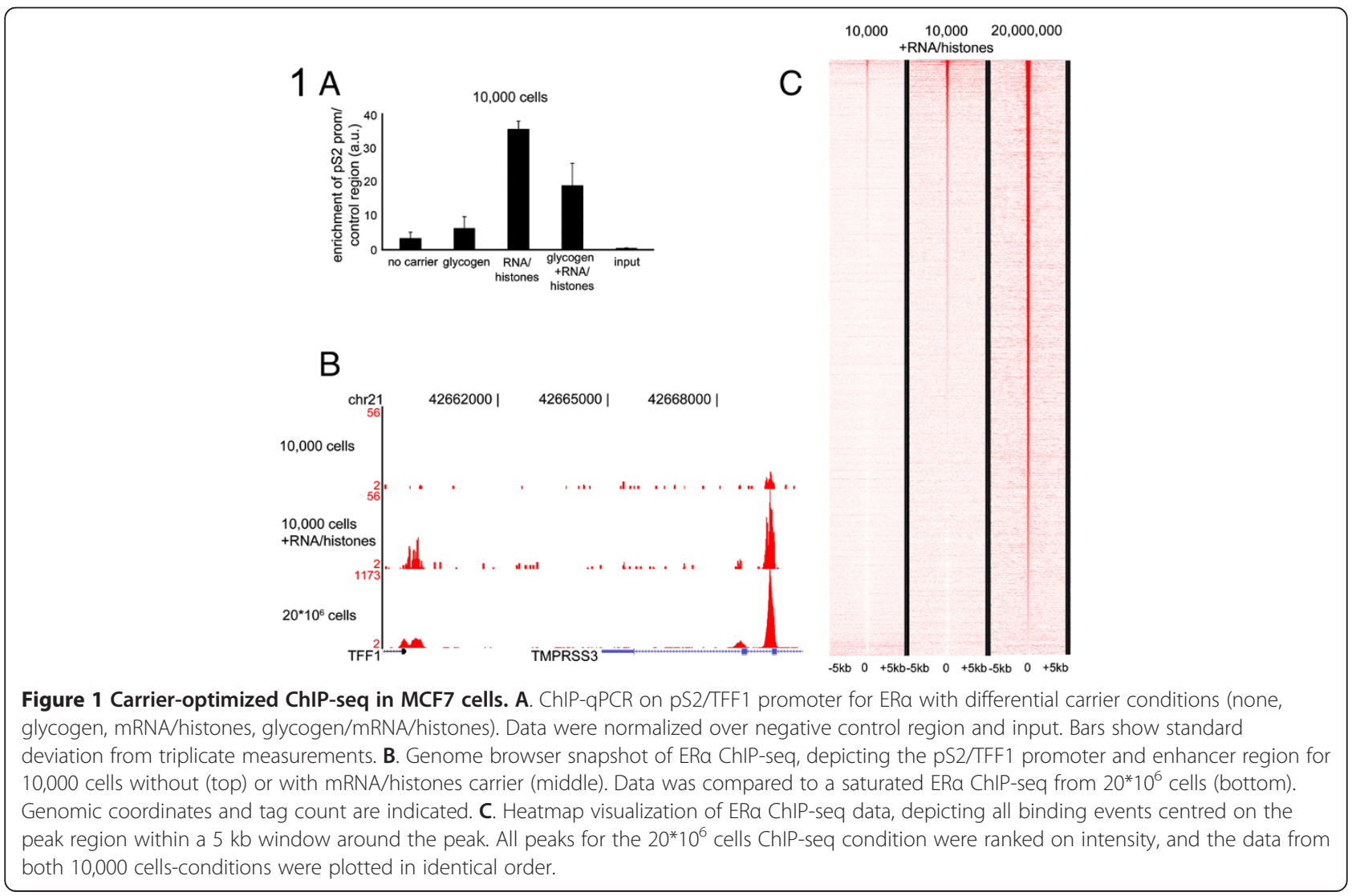


Even though the increased enrichment at the pS2 promoter was indicative of increased ChIP efficiency, this cannot be generalized towards all ER $\alpha /$ chromatin interactions. Therefore, the ER $\alpha$ ChIP-samples were further processed and prepared for sequencing. Only the two extreme conditions (no carrier versus mRNA/Histones carrier) were sequenced and compared to a publicly available dataset of proliferating MCF7 cells, performed on $\sim 20$ million cells [10]. The carrier mRNA and histone were degraded prior to amplification of enriched ChIP DNA. An example of difference in binding capacity in the presence and absence of carrier mRNA/histones is shown in Figure $1 \mathrm{~B}$ and all binding events are illustrated as a heatmap in Figure 1C. Importantly, we ranked all ER $\alpha$ binding peaks observed in the sample with $\sim 20$ million cells and found that $\sim 60 \%$ of these were recapitulated when using the mRNA and histones as carrier from 10,000 cells. This is a substantial improvement compared to ER $\alpha$ ChIP-seq without carrier, where only signal was detected at $\sim 20 \%$ of peaks. Heatmap visualisations and quantifications indicated that peak calling on 10,000 cells with RNA/histones carrier provided comparable signal intensity distributions as the saturated ChIP. This in contrast to the 10,000 cells analysis without carriers (Additional file 2: Figure S2) where mainly background was found. Grouping the ChIP distributions on the basis of signal intensities showed that peaks with high-intensity signal under the RNA/histones carrier ChIP conditions, consistently provided high signal distributions for the saturated ChIP, and vice versa (Additional file 3: Figure S3). No subsets of false-positive and false-negative regions were observed (Additional file 3: Figure S3). Interestingly, the dynamic range of signal intensity as detected in the saturated ChIP was dampened under the carrier conditions (Additional file 3: Figure S3A). Cumulatively, these data show that the addition of carrier chromatin facilitates transcription factor ChIP-seq from 10,000 cells, without negatively effecting the amplification or global fidelity of the process.

In order to confirm the biological validity of the ER $\alpha$ binding events mapped using mRNA/histone as carrier for ChIP-seq from 10,000 cells, we integrated the binding information with estrogen (E2)-mediated gene expression profiles. For all E2-regulated genes, we determined whether our altered ChIP protocol enabled the identification of proximal ER $\alpha$ as compared to the saturated ChIP (Figure 2). We used a publically available gene expression microarray dataset, from MCF7 cells treated for 6 hours with E2 [13] and found 1,821 transcripts up-regulated and 1,812 down-regulated by E2. A window of $20 \mathrm{~kb}$ around the transcription start-site was applied to determine proximal ER $\alpha$ binding (which has previously been shown to be the optimal window between ER $\alpha$ binding events and promoters of target genes [12]). For the saturated ChIP-seq, approximately $60 \%$ of all E2 up- and downregulated genes had a proximal ER $\alpha$ binding event (Figure 2A), which is consistent with our previous report [13]. Using the ER $\alpha$ binding events discovered from 10,000 cells with the mRNA/histones as carrier, we found that $38 \%$ of the E2 upregulated genes had an adjacent $\mathrm{ER} \alpha$ binding event (Figure 2A), a figure under-represented because of the fewer ER $\alpha$ binding events in this category. We also ranked the strongest gene-proximal ER $\alpha$ binding events from the mRNA/histone carrier-sample based on tag count, resulting in binding at well-described ER $\alpha$ target genes, including TFF1, RARA, GREB1 and XBP1 (Figure 2B).

\section{Carrier-ChIP from core needle biopsies of human breast tumours}

Given the findings that mRNA/histone carrier appeared to assist ChIP-seq from limited cell numbers, we applied this approach to clinically relevant samples. We chose to study core needle biopsy samples from breast cancer patients, since they represent clinically available, but limited material that would be appropriate for our approach. These samples were all positive for Estrogen Receptor, as determined by immunohistochemistry (Additional file 4: Table S2). We tested the two carrier conditions independently; 1 . glycogen 2 . mRNA in combination with recombinant Histone 2B. ER $\alpha$ ChIP-seq was performed in all core needle biopsies. When using glycogen as a carrier, a small level of enrichment was observed from the biopsy samples, as exemplified at multiple binding sites (Figure 3A). Importantly, in the presence of mRNA/histone carriers, the enrichment at both the example regions and globally, was significantly increased. This resulted in $669 \mathrm{ER} \alpha$ peaks in the presence of glycogen and 3,366 peaks in the presence of mRNA/histone carrier for two biopsies with directly comparable clinical parameters (Additional file 4: Table $\mathrm{S} 2$ ). Importantly, in the presence of mRNA/histone carrier, ER $\alpha$ ChIP-seq signal was comparable to the level observed in the cell line (Figure 3A), suggesting that the presence of the carrier enables increased ER $\alpha$ ChIP-seq signal, even when applied to limited samples obtained from core needle biopsies.

$\mathrm{ER} \alpha$ binding events are rarely found at promoters (5\%) and the majority of binding sites is normally enriched at introns and distal intergenic regions [18]. We analysed the binding site localizations and found that the mRNA/ histones assisted ChIP-seq showed comparable genomic distributions compared to cell line data (Figure 3B). Motif analyses were performed for the mRNA/histone assisted ChIP condition (Figure 3C). As expected, ER DNA binding motifs were the most strongly enriched motifs.

Since ER $\alpha$ is the driving transcription factor in luminal breast cancer, we interrogated the genes proximal to the 


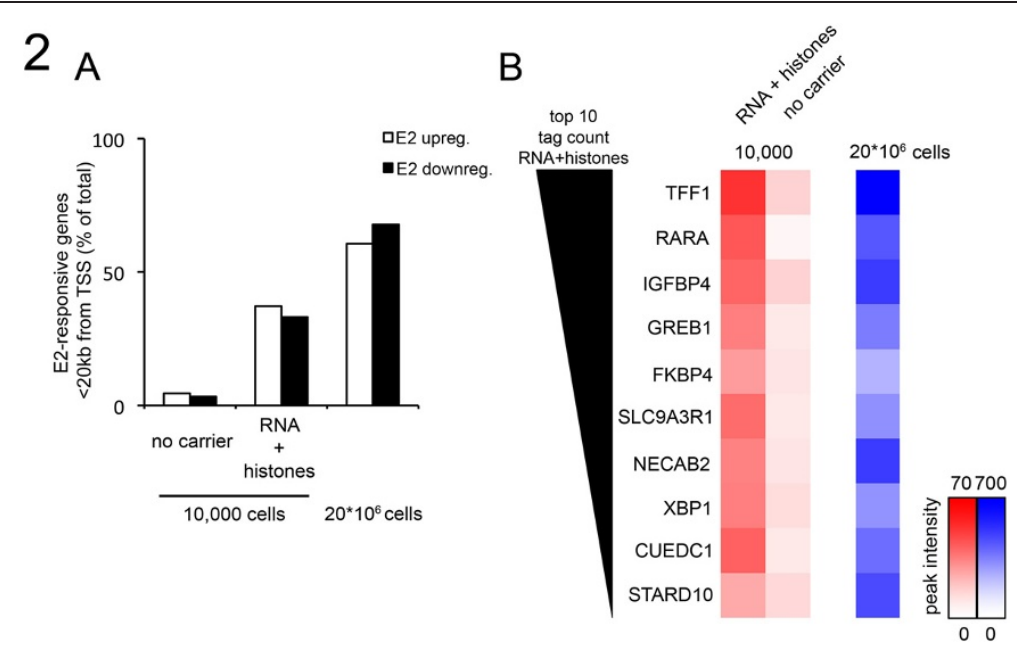

Figure 2 Proximal Estradiol-responsive genes and carrier ERa ChIP-seq enrichment. A. Estradiol up- (white) and down-regulated (black) genes were interrogated for proximal ERa binding events under saturated conditions with $20^{*} 10^{6}$ cells (right), as well as ChIP-seq on 10,000 cells without (left) and with mRNA/histones carrier (middle). The percentage of E2-reponsive genes with a proximal ERa binding site is shown. B. ERa binding events with mRNA/histones carriers were ranked and proximal E2-upregulated genes were determined for the top 10 highest intensity binding sites. Heatmap indicates tag count.

ER $\alpha$ binding events, and determined whether these were previously identified as basal or luminal gene signatures in independent cohorts of breast cancer patients [2] (Figure 3D). For the glycogen carrier condition, none of these hallmark genes were found to have a proximal $E R \alpha$ binding event for any of the tumour samples tested (data not shown). For the mRNA/histone-assisted condition, an enrichment of luminal genes was found over basal-signature genes (Figure 3D). Expression of these signature genes with proximal ER $\alpha$ binding events was found to correlate with a favourable outcome after endocrine treatment in a meta-analysis of breast cancer patients with ER $\alpha$-positive disease [19] (Figure 3E), indicating that our carrier ChIP-seq protocol enabled us to identify direct $\mathrm{ER} \alpha$ target genes with clinical implications from core needle biopsy samples.

\section{Conclusion}

The genome-wide assessment of ER $\alpha /$ chromatin interactions has been performed in tissue culture cell lines [18] and primary breast cancer tissue [11]. Even though ChIP-seq is a very powerful technology to delineate transcription factor-chromatin interactions and to identify specific locations of histone marks on a genome-wide level, the technology does have its intrinsic limitations. The availability of a specific antibody and applicable for immunoprecipitation from formaldehyde-fixed material are major limitations. In addition, there are potential issues related to epitope masking. Another major limitation of ChIP-seq analyses is the need for a certain amount of starting material. Reports have described ChIP-seq analyses on histone marks on limited starting material [14], but these represent relatively stable covalent interactions, as compared to the dynamic and unstable interactions between transcription factors and chromatin. In directed ChIP analyses, the benefit of carrier during the immunoprecipitation procedure has proven to be substantial [16]. In that method, nonrelevant chromatin (e.g. drosophila DNA), is added as a carrier during the procedure, in order to enhance the enrichment from the limited but relevant cellular material. While this approach can be applied for qPCR-ChIP, it is not readily applicable for ChIP-seq, which will sequence all DNA, regardless of the species, resulting in substantial dilution of the relevant enriched DNA. How the addition of the carriers exactly enhances the ChIP procedure on a molecular level still needs to be resolved. Most likely, the bulky material of the carrier helps to retain the small amounts of relevant chromatin throughout the isolation procedure. In addition, it might prove an additional benefit of blocking nonspecific antibody/protein interactions, thereby increasing substrate specificity of the antibody in the reaction to reduce background signal.

Here, we introduce a novel mode of carrier-assisted Chromatin Immunoprecipitation that can be applied to transcription factor mapping by ChIP-seq. The enrichment from limited cellular material is enhanced by the addition of mRNA and recombinant histone protein, which function as a carrier. Importantly, both components of the carrier can subsequently be removed and provide an approach that is cost effective, easily applied and applicable in next-generation sequencing reactions.

We applied this approach to map ER $\alpha$ in 10,000 asynchronous MCF7 cells, which results in an improved 


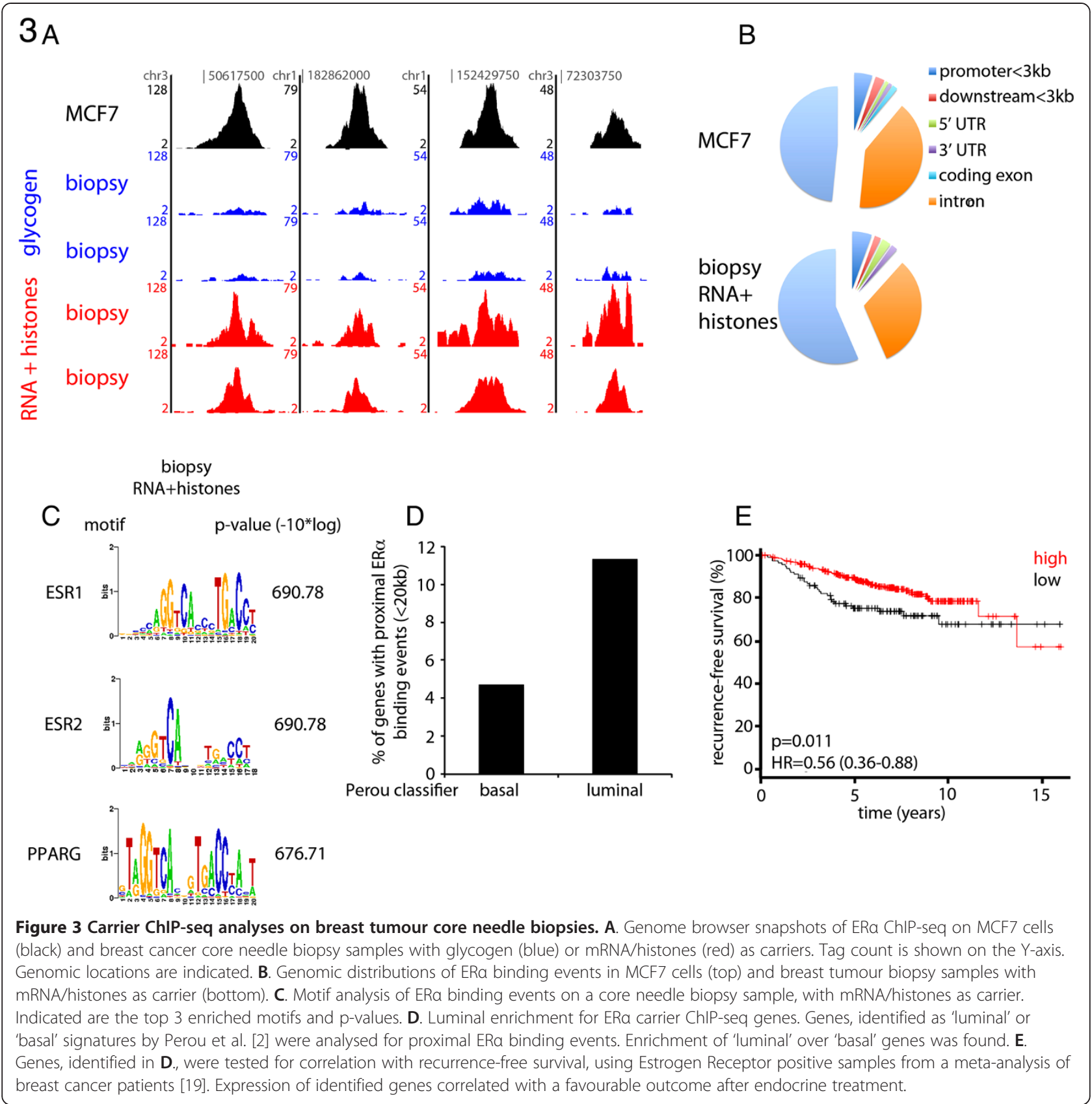

DNA recovery, when compared to no carrier. Although this recapitulated $\sim 60 \%$ of the signal observed when performing ER $\alpha$ ChIP-seq in saturating numbers of cells, these possessed all the expected characteristics of robust ER $\alpha$ binding events. The dynamic range of the ChIP signal intensity was dampened under carrier conditions, possibly implying that weaker ChIP enrichment regions are enhanced in our method. Furthermore, as a proof-ofprinciple, we applied our carrier-assisted ChIP protocol to limited material derived from core needle biopsy samples, isolated from breast cancer patients. Again, the presence of the mRNA/histone carrier resulted in increased ER $\alpha$ ChIP-seq signal, allowing for discovery of clinically and biologically relevant ER $\alpha$ regulatory domains from these samples. Several approaches have been recently described $[15,20]$ that enhance amplification from limited DNA. Although these are powerful methods, they assume that there is sufficient DNA enriched by ChIP, but currently, methods do not exist that enable the ChIP enrichment from limited material for integration with these novel amplification approaches. We believe that our modified carrier ChIP-method improves the ability for purifying transcription factorassociated DNA from limited material, such as tumour 
samples. The potential exists for coupling our modified ChIP approach with the novel amplification methods for greater ChIP-seq fidelity and signal from primary material, potentially permitting high-resolution transcription factor mapping even from clinical samples.

\section{Methods}

\section{Chromatin Immunoprecipitations}

ChIP was performed as described before [17], but with a number of modifications. During the Immunoprecipitation, $100 \mu \mathrm{g} / \mathrm{ml}$ glycogen (Invitrogen) or $20 \mu \mathrm{g} /$ ml recombinant histone 2B (M2505S; New England Biolabs) and $1 \mu \mathrm{g} / \mathrm{ml}$ human mRNA (Invitrogen) was added as carriers for the ChIP. During the entire procedure, non-sticky eppendorf tubes were used (13-698794; Fisher Scientific). $5 \mu \mathrm{g}$ antibody used was raised against ER $\alpha$ (SC-543; Santa Cruz), conjugated to $50 \mu \mathrm{l}$ protein A Dynabeads (Invitrogen). After immunoprecipitation, ten RIPA washes were performed (samples transferred to a fresh eppendorf during the last wash), followed by one TBS wash and reverse crosslinking, as described [17].

\section{Solexa sequencing}

ChIP DNA was amplified as described [17], using 18 PCR amplification cycles. Sequences generated by the Illumina GAIIx genome analyzer (using 36-bp reads) were aligned against NCBI Build 36.3 of the human genome using MAQ (http://maq.sourceforge.net/) with default parameters. Peaks were called using model-based analysis for ChIP-Seq (MACS)[21] run using default parameters. Read counts and duplication rates for all cell line and tumor samples are shown in Additional file 5: Table S1. The number of clonal reads in the carrier sample was higher and may be a result of increased duplication reads of remaining non-ligated adapters.

\section{Motif analysis, heatmaps and genomic distributions of binding events}

ChIP-seq data snapshots were generated using the Integrative Genome Viewer IGV 2.1 (www.broadinstitute. org/igv/). Motif analyses were performed through the Cistrome (cistrome.org), applying the SeqPos motif tool [22]. The genomic distributions of binding sites were analysed using the cis-regulatory element annotation system (CEAS) [23]. The genes closest to the binding site on both strands were analysed. If the binding region is within a gene, CEAS software indicates whether it is in a $5^{\prime}$ UTR, a $3^{\prime} \mathrm{UTR}$, a coding exon, or an intron. Promoter is defined as $3 \mathrm{~kb}$ upstream from RefSeq $5^{\prime}$ start. If a binding site is $>3 \mathrm{~kb}$ away from the RefSeq transcription start site, it is considered distal intergenic.

\section{Tumour tissue handling}

Tissue for ChIP-seq analyses was from four newly diagnosed breast cancer patients who were treated at the Netherlands Cancer Institute. Three specimens were collected under a prospective pre-operative endocrine therapy study approved by the institutional ethics committee. These patients provided written informed consent. Also one sample, taken from anonymous left over material, which would be discarded otherwise, was used. Since we are using anonymous, coded leftover material which is not traced back to the patients and does therefore not interfere with care and/ or prognosis, no ethical approval is required according to Dutch legislation [the Medical Research Involving Human Subjects Act; http://www.ccmo-online.nl/ main.asp?pid=10\&sid=30\&ssid=51) and our institutional medical ethical review board. This conforms as described [24]. Three biopsies (14-gauge, $15 \mu \mathrm{m}$ thick) were taken from each tumour, two of which where frozen with liquid nitrogen and one was formalin-fixed for direct clinical assessment. Tumour cell percentage was determined using Hematoxylin and Eosin (H\&E), and tissue was only used for ChIP-seq analysis when tumour cell percentage was higher then $50 \%$. All samples stained positive for Estrogen Receptor. Further information on the tumor samples and patient characteristics can be found in Additional file 4: Table S2.

\section{Gene expression analyses and survival data}

Genes with an ER $\alpha$ binding site within $20 \mathrm{~kb}$ from the transcription start site were interrogated. Estradiolresponsive genes were used from a publically available database [13]. Luminal and basal genes were previously identified [2]. For survival analyses, a publically available meta-analysis was used. ER $\alpha$ positive-tumours were selected from breast cancer patients who received adjuvant endocrine treatment [19].

\section{Data deposition}

All genomic data is deposited at ArrayExpress under the accession number E-MTAB-1534.

\section{Additional files}

Additional file 1: Figure S1. Carrier ChIP QPCR enrichment for the pS2 promoter and negative control region. ChIP enrichment was calculated as percentage over input, both for the pS2-positive control (left panel) and for the negative control region (right panel). The RNA/histones carrier increases signal as the pS2 positive control region, while it diminishes signal at the negative control site.

Additional file 2: Figure S2. Heatmap visualizations and signal quantifications for the three ChIP conditions. MACS peak caller was applied for sequencing data from samples without carrier $(\mathbf{A})$, with the $\mathrm{RNA}$ /histones carriers $(\mathbf{B})$ and the saturated $\mathrm{ChIP}(\mathbf{C})$. For each peak calling dataset, the corresponding genomic locations were tested for all three sequencing runs, as visualized in heatmaps (arrow head indicated centre of the peak, scale $=5 \mathrm{~kb}$ ) and quantified in the $2 \mathrm{D}$ graphs. 
Additional file 3: Figure S3. Peak subgroups and correlations between carriers/saturated ChIP samples. MACS peak caller was applied for sequencing data from samples with the RNA/histones carriers (A) and the saturated ChIP (B). Peaks were subgrouped in 'high' (I), 'medium' (II) and 'low' (III), and raw signal intensity for the RNA/histones carriers and the saturated ChIP sequencing runs was analyzed. For each of the subsets, the corresponding genomic locations were visualized in heatmaps (arrow head indicated centre of the peak, scale $=5 \mathrm{~kb}$ ) and quantified in the $2 \mathrm{D}$ graphs.

Additional file 4: Table S2. Tumor characteristics, hormonal status and menopausal state.

Additional file 5: Table S1. Sequencing reads and duplication rate.

\section{Competing interests}

The authors declare no competing financial interests.

\section{Authors' contributions}

WZ carried out the experiments and analyzed the data. RK provided the tissue samples and participated in the design of the study. SL participated in the design of the study, coordinated the clinical aspects of the study and helped to draft the manuscript. ER provided the tissue samples and helped in design the clinical aspects of the study. JW performed the pathological assessment of the tissue samples and helped drafting the manuscript. JC and WZ conceived, designed and coordinated the study and drafted the manuscript. All authors read and approved the final manuscript.

\section{Acknowledgements}

We thank James Hadfield for Solexa sequencing, Gordon Brown, Rory Stark and Suraj Menon for bioinformatic help. We thank Sara Dietz for help on the initial development of the protocol.

\section{Funding}

We acknowledge the support of The University of Cambridge, Cancer Research UK, the Dutch Cancer Society, A Sisters Hope and The Netherlands Cancer Institute. J.S.C. is supported by an ERC Starting Grant (242664) and W. Z. is supported by a KWF Dutch Cancer Society fellowship.

\section{Author details}

'Department of Molecular Pathology, The Netherlands Cancer Institute, Amsterdam, the Netherlands. ${ }^{2}$ Department of Pathology, The Netherlands Cancer Institute, Amsterdam, the Netherlands. ${ }^{3}$ Department of Surgical Oncology, The Netherlands Cancer Institute, Amsterdam, the Netherlands. ${ }^{4}$ Department of Medical Oncology, The Netherlands Cancer Institute, Amsterdam, the Netherlands. ${ }^{5}$ Cancer Research UK, Cambridge Research Institute, Li Ka Shing Centre, Robinson Way, Cambridge CB2 ORE, UK. ${ }^{6}$ Department of Oncology, University of Cambridge, Cambridge CB2 OXY, UK.

Received: 5 September 2012 Accepted: 22 March 2013

Published: 8 April 2013

\section{References}

1. Ferlay J, Shin HR, Bray F, Forman D, Mathers C, Parkin DM: Estimates of worldwide burden of cancer in 2008: GLOBOCAN 2008. International journal of cancer Journal international du cancer 2010, 127(12):2893-2917.

2. Perou CM, Sorlie T, Eisen MB, van de Rijn M, Jeffrey SS, Rees CA, Pollack JR, Ross DT, Johnsen H, Akslen LA, et al: Molecular portraits of human breast tumours. Nature 2000, 406(6797):747-752.

3. Osborne CK, Bardou V, Hopp TA, Chamness GC, Hilsenbeck SG, Fuqua SA, Wong J, Allred DC, Clark GM, Schiff R: Role of the estrogen receptor coactivator AIB1 (SRC-3) and HER-2/neu in tamoxifen resistance in breast cancer. J Natl Cancer Inst 2003, 95(5):353-361.

4. Holm C, Rayala S, Jirstrom K, Stal O, Kumar R, Landberg G: Association between Pak1 expression and subcellular localization and tamoxifen resistance in breast cancer patients. J Natl Cancer Inst 2006, 98(10):671-680.

5. Michalides R, Griekspoor A, Balkenende A, Verwoerd D, Janssen L, Jalink K, Floore A, Velds A, van't Veer L, Neefjes J: Tamoxifen resistance by a conformational arrest of the estrogen receptor alpha after PKA activation in breast cancer. Cancer Cell 2004, 5(6):597-605.

6. Redmond AM, Bane FT, Stafford AT, Mcllroy M, Dillon MF, Crotty TB, Hill AD, Young LS: Coassociation of estrogen receptor and $\mathrm{p} 160$ proteins predicts resistance to endocrine treatment; SRC-1 is an independent predictor of breast cancer recurrence. Clinical cancer research: an official journal of the American Association for Cancer Research 2009, 15(6):2098-2106.

7. Shou J, Massarweh S, Osborne CK, Wakeling AE, Ali S, Weiss H, Schiff R: Mechanisms of tamoxifen resistance: increased estrogen receptor-HER2 /neu cross-talk in ER/HER2-positive breast cancer. J Nat/ Cancer Inst 2004 96(12):926-935.

8. Molina MA, Codony-Servat J, Albanell J, Rojo F, Arribas J, Baselga J: Trastuzumab (herceptin), a humanized anti-Her2 receptor monoclonal antibody, inhibits basal and activated Her2 ectodomain cleavage in breast cancer cells. Cancer Res 2001, 61(12):4744-4749.

9. Lupien M, Meyer CA, Bailey ST, Eeckhoute J, Cook J, Westerling T, Zhang X Carroll JS, Rhodes DR, Liu XS, et al: Growth factor stimulation induces a distinct ER(alpha) cistrome underlying breast cancer endocrine resistance. Genes Dev 2010, 24(19):2219-2227.

10. Hurtado A, Holmes KA, Ross-Innes CS, Schmidt D, Carroll JS: FOXA1 is a key determinant of estrogen receptor function and endocrine response. Nat Genet 2011, 43(1):27-33.

11. Ross-Innes CS, Stark R, Teschendorff AE, Holmes KA, Ali HR, Dunning MJ, Brown GD, Gojis O, Ellis IO, Green AR, et al: Differential oestrogen receptor binding is associated with clinical outcome in breast cancer. Nature 2012, 481(7381):389-393

12. Fullwood MJ, Liu MH, Pan YF, Liu J, Xu H, Mohamed YB, Orlov YL, Velkov S Ho A, Mei PH, et al: An oestrogen-receptor-alpha-bound human chromatin interactome. Nature 2009, 462(7269):58-64.

13. Zwart W, Theodorou V, Kok M, Canisius S, Linn S, Carroll JS: Oestrogen receptor-co-factor-chromatin specificity in the transcriptional regulation of breast cancer. EMBO J 2011, 30(23):4764-4776.

14. Adli $M$, Zhu J, Bernstein BE: Genome-wide chromatin maps derived from limited numbers of hematopoietic progenitors. Nat Methods 2010, 7(8):615-618.

15. Shankaranarayanan $P$, Mendoza-Parra MA, Walia M, Wang L, Li N, Trindade LM, Gronemeyer H: Single-tube linear DNA amplification (LinDA) for robust ChIP-seq. Nat Methods 2011, 8(7):565-567.

16. O'Neill LP, VerMilyea MD, Turner BM: Epigenetic characterization of the early embryo with a chromatin immunoprecipitation protocol applicable to small cell populations. Nat Genet 2006, 38(7):835-841.

17. Schmidt D, Wilson MD, Spyrou C, Brown GD, Hadfield J, Odom DT: ChIPseq: using high-throughput sequencing to discover protein-DNA interactions. Methods 2009, 48(3):240-248.

18. Carroll JS, Meyer CA, Song J, Li W, Geistlinger TR, Eeckhoute J, Brodsky AS, Keeton EK, Fertuck KC, Hall GF, et al: Genome-wide analysis of estrogen receptor binding sites. Nat Genet 2006, 38(11):1289-1297.

19. Gyorffy B, Lanczky A, Eklund AC, Denkert C, Budczies J, Li Q, Szallasi Z: An online survival analysis tool to rapidly assess the effect of 22,277 genes on breast cancer prognosis using microarray data of 1,809 patients. Breast Cancer Res Treat 2010, 123(3):725-731.

20. Adli M, Bernstein BE: Whole-genome chromatin profiling from limited numbers of cells using nano-ChIP-seq. Nat Protoc 2011, 6(10):1656-1668.

21. Zhang Y, Liu T, Meyer CA, Eeckhoute J, Johnson DS, Bernstein BE, Nusbaum C, Myers RM, Brown M, Li W, et al: Model-based analysis of ChIP-Seq (MACS). Genome Biol 2008, 9(9):R137.

22. He HH, Meyer CA, Shin H, Bailey ST, Wei G, Wang Q, Zhang Y, Xu K, Ni M, Lupien $M$, et al: Nucleosome dynamics define transcriptional enhancers. Nat Genet 2010, 42(4):343-347.

23. Ji X, Li W, Song J, Wei L, Liu XS: CEAS: cis-regulatory element annotation system. Nucleic Acids Res 2006, 34(Web Server issue):W551-W554.

24. van Diest PJ: No consent should be needed for using leftover body material for scientific purposes. For. BMJ 2002, 325(7365):648-651.

doi:10.1186/1471-2164-14-232

Cite this article as: Zwart et al.: A carrier-assisted ChIP-seq method for estrogen receptor-chromatin interactions from breast cancer core needle biopsy samples. BMC Genomics 2013 14:232. 